Nanomedicine (Lond). 2012 August ; 7(8): 1197-1209. doi:10.2217/nnm.12.18.

\title{
Silver nanoparticles do not influence stem cell differentiation but cause minimal toxicity
}

\author{
Meghan E Samberg ${ }^{1,2}$, Elizabeth G Loboa ${ }^{2,3}$, Steven J Oldenburg ${ }^{4}$, and Nancy A Monteiro- \\ Riviere ${ }^{1,2,{ }^{*}}$ \\ ${ }^{1}$ Center for Chemical Toxicology Research \& Pharmacokinetics, North Carolina State University, \\ NC, USA \\ ${ }^{2}$ Joint Department of Biomedical Engineering at North Carolina State University \& the University \\ of North Carolina-Chapel Hill, NC, USA \\ ${ }^{3}$ Department of Materials Science \& Engineering, North Carolina State University, NC, USA \\ ${ }^{4}$ NanoComposix, San Diego, CA, USA
}

\section{Abstract}

\begin{abstract}
Aims-To evaluate the toxicity and cellular uptake of both undifferentiated and differentiated human adipose-derived stem cells (hASCs) exposed to silver nanoparticles (Ag-NPs), and to assess their effect on hASC differentiation.
\end{abstract}

Materials \& methods-hASC were exposed to 10- or 20-nm Ag-NPs at concentrations of 0.1, $1.0,10.0,50.0$ and $100.0 \mu \mathrm{g} / \mathrm{ml}$ either before or after differentiation down the adipogenic or osteogenic pathways.

Results-Exposure of hASC to either 10- or 20-nm Ag-NPs resulted in no significant cytotoxicity to hASC, and minimal dose-dependent toxicity to adipogenic and osteogenic cells at $10 \mu \mathrm{g} / \mathrm{ml}$. Each of the hASC, adipogenic and osteogenic cells showed cellular uptake of both 10and 20-nm Ag-NPs, without causing significant ultrastructural alterations. Exposure to 10- or 20$\mathrm{nm}$ Ag-NPs did not influence the differentiation of the cells, and at antimicrobial concentrations of Ag-NPs resulted in a minimal decrease in viability.

Conclusion-The biocompatibility of Ag-NPs with both undifferentiated and differentiated hASC establishes their suitability for incorporation into tissue-engineered graft scaffolds, for the prevention of bacterial contamination upon implantation.

(c) 2012 Future Medicine Ltd

*Author for correspondence: Tel.: +1 919513 6426, Fax: +1 919513 6358, nancy_monteiro@ncsu.edu.

For reprint orders, please contact: reprints@ futuremedicine.com

Ethical conduct of research

The authors state that they have obtained appropriate institutional review board approval or have followed the principles outlined in the Declaration of Helsinki for all human or animal experimental investigations. In addition, for investigations involving human subjects, informed consent has been obtained from the participants involved.

Financial \& competing interests disclosure

This research was supported by the United States Air Force Office of Scientific Research (USAFOSR) grant FA 9550-08-1-0182 and the NIH (RO1 ES016138). S Oldenburg is the President of nanoComposix (CA, USA). Portions of this article were selected for platform presentation in the Nanotoxicology - Nanosilver Particulates platform session at the 50th Annual Meeting for the National Society of Toxicology (Washington, DC, USA, 8 March 2011). The authors have no other relevant affiliations or financial involvement with any organization or entity with a financial interest in or financial conflict with the subject matter or materials discussed in the manuscript apart from those disclosed.

No writing assistance was utilized in the production of this manuscript. 


\section{Keywords}

in vitro; nanoparticles; nanotechnology; nanotoxicology; scaffolds; silver; stem cells

Over 300,000 incidences of surgical-site infection occur annually in the USA, arising in approximately $2-3 \%$ of patients who have undergone surgery. Although rates vary widely according to the type of surgery, $33 \%$ of infections occur from orthopedic surgeries resulting in increased treatment intensity, prolonged length of stay, higher costs and greater risk for readmission or death [101]. The most common bacterial strain responsible for surgical infection is Staphylococcus aureus, and the only method employed currently to avoid infection is through strict adherence to sterile surgical conditions and through the administration of prophylactic antibiotics [1]. However, drug-resistant organisms such as methicillin-resistant $S$. aureus and vancomycin-resistant enterococci are still problematic [1].

Due to the enhanced antibacterial activity of silver (Ag) at the nanoscale level, Agnanoparticles (NPs) are the most used nanomaterial and the most valuable in the medical and personal hygiene fields through their incorporation into hundreds of consumer products [102]. Previous studies have demonstrated that Ag-NPs exert antimicrobial effects on a wide spectrum of bacteria such as Escherichia coli, Enterococcus faecium, Vibrio cholera, $S$. aureus and even methicillin-resistant $S$. aureus [2-4]. Since Ag compounds have been used for centuries with a lack of toxicity or carcinogenicity to the immune, cardiovascular, nervous or reproductive systems, the use of Ag-NPs in products has largely been unregulated [103]. Presently, there is no consensus on the cytotoxicity of Ag-NPs; however, the majority of publications do show reduced cell viability and increased reactive oxygen species generation following Ag-NP exposure [5].

An application for Ag-NPs is their incorporation into implantable materials such as prostheses and skin or bone grafts. In recent years, tissue engineering of patient-specific bone grafts has been used to replace damaged or defective tissues, and has become a viable alternative to autografts and allografts [6]. Advances in human stem cell research demonstrate that these cells have unique properties that are important for the development of engineered tissue constructs, including high proliferation rates and self-renewal capacity, maintenance of the unspecialized state, and specialized differentiation under given conditions. Human mesenchymal stem cells (hMSCs) isolated from either adult bone marrow or adipose tissue possess a high capacity for self-replication and have the potential to differentiate into osteogenic, chondrogenic, adipogenic and tenogenic lineages when placed in an appropriate environment $[7,8]$.

The interaction and metabolism of Ag-NPs with cultured osteoblasts grown from hMSC should be minimal for their incorporation into a scaffold to be successful. A number of studies have shown that various mesoporous silica and magnetic NPs used for cell tracking have no apparent deleterious effects on the morphology, cell proliferation, cell viability and differentiation efficiency of stem cells [9-11]. However, Ag-NPs and Ag ions have been reported to attach to cell membranes, and interact with cellular molecules such as DNA, bacterial cell wall components or sulfhydryl groups of metabolic enzymes [2-4]. Therefore, bacterial replication, membrane permeability, and different metabolic pathways may be interrupted. The objective of this study was to evaluate the toxicity and cellular uptake of both undifferentiated and differentiated human adipose-derived stem cells (hASCs) exposed to Ag-NPs, and to assess their potential effect on hASC differentiation. Understanding the interactions between Ag-NPs and undifferentiated hASC is important to determine whether the antimicrobial activity of Ag-NPs is detrimental to the differentiation of hASC down the adipogenic or osteogenic pathways for tissue engineering scaffold systems. 


\section{Materials \& methods}

\section{Ag-NPs}

Two solutions of spherical Ag-NPs were obtained from NanoComposix (CA, USA), each at Ag-NP concentration of $1.00 \mathrm{mg} / \mathrm{ml}$ with diameters of 10 and $20 \mathrm{~nm}$. Ag-NPs were synthesized by ammonium hydroxide-catalyzed growth of Ag onto 5-nm gold-seed particles. Concentration of the particles was achieved via tangential flow filtration. The Ag-NPs were then serially washed with 20 volume equivalents of phosphate buffer (PB), resuspended in stabilizing citrate buffered deionized (DI) water and stored at $4^{\circ} \mathrm{C}$ in the dark.

Particle size was determined by dynamic light scattering (DLS) and transmission electron microscopy (TEM) to confirm the manufacturer-identified diameters. To measure the hydrodynamic diameters and the $\zeta$-potential, samples of Ag-NPs were suspended at the highest dosing concentration in DI water, immediately placed in a disposable cuvette and analyzed using a Zetasizer Nano-ZS (Malvern Instruments, Inc., Worcestershire, UK). The DLS readings were performed at the standard characterization temperature of $25^{\circ} \mathrm{C}$. Each measurement was repeated five-times, with ten to 20 runs as optimized by the instrument. Data was culled based on the correlogram, size quality report and expert advice rendered by the Dispersion Technology Software (5.03) (Malvern Instruments Inc.). In addition, TEM was utilized to characterize Ag-NP structure, shape and size uniformity. Samples were prepared by placing a drop of homogeneous suspension of each Ag-NP solution at the highest dosing concentration in DI water onto a formvar-coated copper grid and air dried. Samples were examined on an FEI $^{\mathrm{TM}} /$ Philips EM 208S (FEI, OR, USA) TEM operating at an accelerating voltage of $80 \mathrm{kV}$.

\section{hASC isolation \& expansion}

All protocols involving human tissue were approved by the Institutional Review Boards of the University of North Carolina at Chapel Hill (NC, USA) and North Carolina State University (NC, USA). Excess adipose tissue from elective plastic surgery procedures was obtained with donor consent from five premenopausal female donors of Caucasian, Native American or other ethnicities who ranged from 24 to 37 years of age. The hASC were isolated from the adipose tissue by enzymatic digestion using $0.075 \%$ collagenase type I, and characterized as described in $[12,13]$. Cells were seeded into T-75 flasks, and grown to approximately $70-80 \%$ confluency in a $5 \% \mathrm{CO}_{2}$ incubator at $37^{\circ} \mathrm{C}$ for expansion and culture. Cells were harvested using trypsin which increased their passage number incrementally (e.g., passage-0, passage- 1 and so on). At passage-1, hASC from each of the five donors were pooled into a T-75 flask (20,000 cells/donor; 100,000 cells total), expanded and frozen in liquid nitrogen until needed.

Pooled passage- 3 cells were seeded into T-75 cell culture flasks at 100,000 cells/flask, cultured in complete growth medium (minimum essential medium eagle, alpha-modified supplemented with $10 \%$ fetal bovine serum, $2 \mathrm{mM}$ L-glutamine, $100 \mathrm{units} / \mathrm{ml}$ penicillin and $100 \mu \mathrm{g} / \mathrm{ml}$ streptomycin), and maintained at $37^{\circ} \mathrm{C}$ in a humidified atmosphere of $5 \% \mathrm{CO}_{2}$. Once hASC reached $80 \%$ confluency, after 6 days, cultures were washed twice with phosphate buffered saline and cells were retrieved using trypsin. At this point, hASC were plated into 12-well plates (5000 cells/well) to assess viability, into T-25 flasks $(50,000$ cell/ flask) to assess cellular uptake, and into six-well plates (50,000 cells/well) to assess any AgNP effects on differentiation. Differentiation of cells was achieved via hASC culture in osteogenic medium (complete growth medium supplemented with $50 \mu \mathrm{M}$ ascorbic acid, 0.1 $\mu \mathrm{M}$ dexamethasone and $10 \mathrm{mM} \beta$-glycerophosphate), or in adipogenic medium (complete growth medium supplemented with $1 \mu \mathrm{M}$ dexamethasone, $5 \mu \mathrm{g} / \mathrm{ml}$ insulin, $100 \mu \mathrm{M}$ 
indomethacin and $500 \mu \mathrm{M}$ isobutylmethylxanthine), or in complete growth medium to maintain the cells in their progenitor cell state.

\section{Cell viability}

To determine the effect of Ag-NPs on both undifferentiated and differentiated hASC viability, undifferentiated hASC were grown to $100 \%$ confluency in complete growth medium for 4 days. To determine the baseline viability, hASC were first differentiated down the osteogenic and adipogenic pathways or maintained in their proliferative state for 14 days and then exposed for $24 \mathrm{~h}$ to concentrations of $0,0.1,1.0,10.0,50.0$ and $100.0 \mu \mathrm{g} / \mathrm{ml}$ of either 10- or 20-nm Ag-NPs. As a comparison to examine any effect internalized Ag-NPs have on hASC, cells were first exposed for $24 \mathrm{~h}$ to Ag-NPs and then differentiated down the osteogenic or adipogenic pathways (or maintained in their proliferative state) for 14 days. At the conclusion of each of these alternatives, the viability of the hASC was assessed with alamarBlue ${ }^{\circledR}$ (Molecular Probes, Invitrogen, OR, USA) viability assay; this assay was chosen based upon its dye having minimal interactions with Ag-NPs [14]. Briefly, a 10\% alamarBlue solution was added to each well, incubated for $3 \mathrm{~h}$ and fluorescence quantitated on a Spectra Max ${ }^{\circledR}$ Gemini $^{\text {TM }}$ (Molecular Devices, CA, USA) EM spectro photometer at an excitation wavelength of $545 \mathrm{~nm}$ and an emission wavelength of $590 \mathrm{~nm}$. The fluorescence values were normalized to controls and expressed as percent viability.

\section{Cellular uptake}

To view potential cellular uptake of Ag-NPs into hASC, undifferentiated hASC were grown to $100 \%$ confluency in complete growth medium for 5 days. To determine baseline uptake data, hASC were first differentiated down the osteogenic or adipogenic pathways or maintained in their proliferative state for 14 days and then exposed for $24 \mathrm{~h}$ to concentrations of $0,0.1,1.0,10.0,50.0$ and $100.0 \mu \mathrm{g} / \mathrm{ml}$ of either 10- or 20-nm Ag-NPs. As a comparison to examine any morphological and ultrastructural effect internalized Ag-NPs have on hASC, cells were first exposed for $24 \mathrm{~h}$ to Ag-NPs and then differentiated down the osteogenic or adipogenic pathways (or maintained in their proliferative state) for 14 days. At the conclusion of each of these alternatives, cells were harvested with trypsin, rinsed in Hank's balanced salt solution and fixed for $24 \mathrm{~h}$ in Trump's fixative at $4^{\circ} \mathrm{C}$. Cells were rinsed in $0.1 \mathrm{M} \mathrm{PB}$ ( $\mathrm{pH} 7.2$ ), pelleted in a microfuge tube, resuspended and quickly pelleted in 3\% molten agar. Samples were postfixed in $1 \%$ osmium tetroxide in $0.1 \mathrm{M} \mathrm{PB}$ for $1 \mathrm{~h}$ at $4^{\circ} \mathrm{C}$, washed with DI water, dehydrated through an ascending ethanol concentration, cleared in acetone, infiltrated and embedded with Spurr's resin, and polymerized at $70^{\circ} \mathrm{C}$ overnight. Cells were not stained to allow for better visualization of the Ag-NPs and to ensure the absence of stain precipitate artifacts. TEM analysis was performed to determine if any morphological or ultrastructural changes occurred between untreated cells and cells exposed to Ag-NPs either prior to or following differentiation, as well as any abnormalities in intracellular lipid accumulation within adipogenic cells or extracellular calcium accretion by osteogenic cells.

\section{Effects on differentiation}

To assess any effects of Ag-NPs on hASC differentiation, undifferentiated hASC were grown to $100 \%$ confluency in complete growth medium for 5 days. Then, hASC were exposed for $24 \mathrm{~h}$ to either 10- or 20-nm Ag-NPs at concentrations of 0, 0.1, 1.0, 10.0,50.0 and $100.0 \mu \mathrm{g} / \mathrm{ml}$, made fresh in complete growth medium. After $24 \mathrm{~h}$ the medium was removed, and cells were differentiated down the osteogenic or adipogenic pathways or maintained in their proliferative state for 14 days. Osteogenic differentiation was determined with Alizarin Red S staining for extracellular calcium accretion, and adipogenic differentiation was determined with Oil Red O staining for intercellular lipid accumulation. To assess differences in staining between control and treatment wells, digital light 
microscopy images were obtained from each well ( $\mathrm{n}=6$ wells per treatment), and the pixel area per image (three images per well) covered by the stained calcium or lipid was determined by color-based image analysis using Adobe ${ }^{\circledR}$ Photoshop ${ }^{\circledR}$.

\section{Statistical analysis}

The mean values for hASC percent viability (normalized to control) and pixel area per image for each treatment were calculated, and the significant differences $(\mathrm{p}<0.05)$ determined by the PROC GLM Procedure (SAS 9.1 for Windows; SAS Institute, NC, USA). When significant differences were found, multiple comparisons were performed with the Tukey's Studentized Range HSD test at $p<0.05$ level of significance. The Dunnett's t-test was performed to determine the significance at $\mathrm{p}<0.05$ of differences between control and treatment groups. Data were expressed as the mean \pm standard error of the mean of two plates ( $n=6$ wells per treatment) and three images per treatment.

\section{Results}

\section{Ag-NP characterization}

The physicochemical properties of the Ag-NPs can be found in Table 1, which lists the physical and hydrodynamic diameters of the Ag-NPs according to TEM and DLS, respectively, as well as the supplied Ag-NP solution concentration, particle concentration and $\zeta$-potential. As is typical for nanomaterials, the hydrodynamic diameter of the 10- and 20-nm Ag-NPs measured by DLS was found to be slightly larger (21.7 and $27.4 \mathrm{~nm}$, respectively) than the physical diameter measured by TEM (7.2 and $19.2 \mathrm{~nm}$, respectively). The significantly larger hydrodynamic diameter of the 10-nm Ag-NPs is most likely attributable to the formation of small, loosely bound Ag-NP dimers. The $\zeta$-potential of a colloid characterizes its stability and ability to resist aggregation, with greater $\zeta$-potentials having greater stability. Both the 10- and 20-nm Ag-NPs had $\zeta$-potentials between -40 and $-50 \mathrm{eV}$, which indicated good stability. The stability and lack of aggregated clusters is largely due to the addition of stabilizing citrate buffer. According to TEM, both 10- and 20$\mathrm{nm} \mathrm{Ag-NPs} \mathrm{were} \mathrm{found} \mathrm{to} \mathrm{be} \mathrm{spherical} \mathrm{in} \mathrm{shape} \mathrm{and} \mathrm{highly} \mathrm{uniform} \mathrm{in} \mathrm{size} \mathrm{(Figure} \mathrm{1).}$

\section{Cell viability}

When undifferentiated hASC were exposed to 10- and 20-nm Ag-NPs for $24 \mathrm{~h}$, and then differentiated for 14 days, no significant decrease in viability was observed for the undifferentiated hASC. A concentration-dependent toxicity was observed for both osteogenic and adipogenic cells starting at an Ag-NP concentration of $10 \mu \mathrm{g} / \mathrm{ml}$ (Figure 2A $\&$ B). Similarly, the addition of either 10- or 20-nm Ag-NPs following hASC differentiation resulted in no significant decrease in viability of undifferentiated hASC at any concentration, while concentration-dependent toxicity was observed for 10-nm Ag-NPs at $100 \mu \mathrm{g} / \mathrm{ml}$ for osteogenic cells and at $10 \mu \mathrm{g} / \mathrm{ml}$ for adipogenic cells (Figure 2C), and for 20$\mathrm{nm}$ Ag-NPs at $50 \mu \mathrm{g} / \mathrm{ml}$ for osteogenic cells and at $100 \mu \mathrm{g} / \mathrm{ml}$ for adipogenic cells (Figure $2 \mathrm{D})$. It should be noted that although significant decreases in viability were observed, the maximal loss of viability was only $30 \%$.

\section{Cellular uptake}

Exposure of 10- and 20-nm Ag-NPs to differentiated hASC served as the control for the evaluation of 10- and 20-nm Ag-NP exposure to undifferentiated hASC. Any morphological and ultrastructural changes in cells, as well as changes in intracellular lipid accumulation in adipogenic cells and extracellular calcium accretion in osteogenic cells, were compared with untreated cells. It was observed that for each treatment group, approximately $50 \%$ of all cells contained Ag-NPs within their cytoplasmic vacuoles, and an increase in treatment 
concentration resulted in an increase in Ag-NP agglomerates within the vacuoles. Transmission electron micrographs of cells exposed to $10 \mu \mathrm{g} / \mathrm{ml}$ are shown as a representative sample (Figures 3-5).

Neither 10- nor 20-nm Ag-NP exposure to either undifferentiated or differentiated hASC resulted in any morphological abnormalities to cells compared with controls. Control hASC were slightly elongated and displayed indented nuclei, a prominent nucleolus and small intra-cytoplasmic vacuoles along the periphery of the nucleus (Figure 3A). Small agglomerates of 10-nm Ag-NPs were observed at low magnification within cytoplasmic vacuoles of hASC (Figure 3B), and high magnification of these vacuoles confirmed the presence of Ag-NPs through the highly uniform shape and size of the Ag-NPs (Figure 3C). Similarly, 20-nm Ag-NPs were seen in small agglomerates within vacuoles along the periphery of the nucleus (Figure 3D). High magnification confirmed numerous Ag-NPs within these vacuoles (Figure 3E).

Both 10- and 20-nm Ag-NP exposure to hASC prior to, or following, adipogenic differentiation did not result in any morphological or ultrastructural changes to cells compared with controls. Control adipogenic cells exhibited a more globular morphology with extensive lipid droplet accumulation within the cell (Figure 4A). Small agglomerates of 10-nm Ag-NPs were observed at low magnification within cytoplasmic vacuoles of unstained adipogenic cells, and Ag-NPs were not observed within the lipid droplets (Figure 4B). High magnification of these vacuoles confirmed the presence of Ag-NPs through the highly uniform shape and size of the Ag-NPs (Figure 4C). Similarly, 20-nm Ag-NPs were seen in small agglomerates within cytoplasmic vacuoles (Figure 4D), and high magnification again confirmed Ag-NPs within these vacuoles (Figure 4E).

Both 10- and 20-nm Ag-NP exposure to hASC prior to, or following, osteogenic differentiation did not result in any morphological changes to the cells compared with controls. Control osteogenic cells were more fusiform (Figure 5A), with extensive extracellular calcium accretion (Figure 5B). Numerous small agglomerates of 10-nm AgNPs were observed at low magnification within cytoplasmic vacuoles of the osteogenic cells (Figure 5C), and high magnification of these vacuoles confirmed the presence of Ag-NPs through the highly uniform shape and size of the Ag-NPs (Figure 5D). Similarly, 20-nm AgNPs were seen in small agglomerates within vacuoles (Figure 5E), and high magnification confirmed Ag-NPs within these vacuoles (Figure 5F).

\section{Effects on differentiation}

Exposure of undifferentiated hASC to either 10 or 20-nm Ag-NPs for $24 \mathrm{~h}$ at any concentration did not influence hASC differentiation. Light micrographs of cells exposed to $10 \mu \mathrm{g} / \mathrm{ml}$ are shown as a representative sample (Figures 6-8).

Undifferentiated hASC were stained for calcium accretion with Alizarin Red S (Figure 6AC), and lipid accumulation with Oil Red O (Figure 6D-F). Since the hASC were maintained in complete growth medium, control cells stained for neither calcium (Figure 6A) nor lipid (Figure 6D). There was no significant occurrence of either lipid or calcium following exposure to 10-nm Ag-NPs at any of the concentrations as evidenced by a lack of calcium and lipid staining (Figures 6B \& E). These results were consistent for 20-nm Ag-NPs (Figures 6C \& F).

Adipogenic cells were stained for calcium accretion with Alizarin Red S (Figures 7A-C), and lipid accumulation with Oil Red O (Figures 7D-F). Control adipogenic cells did not stain for calcium (Figure 7A) and stained strongly for lipid (Figure 7D). There was no significant change in lipid accumulation or calcium accretion following exposure to 10-nm 
Ag-NPs at any concentration as evidenced by the continued lack of calcium staining (Figure 7B) and continued strong lipid staining (Figure 7E). Similarly, exposure to 20-nm Ag-NPs at any concentration did not influence the differentiation down the adipogenic pathway, as evidenced by the continued lack of calcium staining (Figure 7C), and continued strong lipid staining (Figure 7F).

Osteogenic cells were stained for calcium accretion with Alizarin Red S (Figures 8A-C), and lipid accumulation with Oil Red O (Figures 8D-F). Control osteogenic cells stained strongly for calcium (Figure 8A) and did not stain for lipid (Figure 8D). There was no significant change in lipid accumulation or calcium accretion following exposure to 10-nm Ag-NPs at any concentration as evidenced by the continued strong calcium staining (Figure 8B) and continued lack of lipid staining (Figure 8E). Similarly, exposure to 20-nm Ag-NPs at any concentration did not influence the differentiation down the osteogenic pathway, as evidenced by the continued strong calcium staining (Figure 8C), and continued lack of lipid staining (Figure 8F).

\section{Discussion}

Bone grafts are the second most transplanted material, second only to blood transfusions [15]. Currently, conventional treatment methods for bone replacement are limited to autografts (grafts derived from the patient), and allografts (grafts derived from a donor). While autografts are the most desirable option due to the significantly reduced immune reaction, patients possess a relative lack of supply. The use of cadaverous allografts provides a larger tissue supply but may cause immunological reactions. Alternatively, hASC are capable of providing patient-specific cells for tissue regeneration therapies due to their high proliferation rates and self-renewal capacity, maintenance of the unspecialized state, migration capability and specialized differentiation into various cell types such as bone, cartilage, fat and muscle. This differentiation may be achieved through chemical induction; however, differentiation may also be influenced by the material properties of the tissueengineered scaffolds onto which they are seeded.

Every year approximately 300,000 patients who have undergone surgery suffer from surgical-site infection [101]. Studies investigating the effects of surgical-site infection have estimated that an infection increases patient stay by an additional 5 days at a cost of nearly US $\$ 18,000$ per patient, bringing the yearly cost of surgical-site infection patient care to over $\$ 10$ billion [101]. Therefore, while the development of tissue engineering of patient-specific bone grafts have become a viable alternative to autografts and allografts, the implantation of these tissue grafts would still suffer from the high rate of surgical-site infection. To overcome this, once an optimal scaffolding material is achieved for each tissue type, antimicrobial activity to prevent infection could be achieved through the incorporation of Ag-NPs. The indiscriminate activity of Ag-NPs and the released ions would provide broadspectrum antimicrobial activity to scaffolding, and decrease the rate of incidence of infection while improving the success rate and ultimate feasibility of tissue-engineered grafts. In addition, this would help to decrease the widespread use of prophylactic antibiotics that has increased the occurrence of bacterial resistance to typical antibiotics such as methicillin. Therefore, the interaction and metabolism of Ag-NPs with hASC should be minimal for their incorporation into a scaffold to be successful. However, the biocompatibility of AgNPs with hASC is a prerequisite to their incorporation into scaffolding. Therefore, this study validated the use of $\mathrm{Ag}$-NP incorporation into a tissue-engineered scaffold by assessing the toxicity, cellular uptake and effect on hASC differentiation following exposure to either 10or 20-nm Ag-NPs at concentrations of 0.1, 1.0, 10.0, 50.0 and $100.0 \mu \mathrm{g} / \mathrm{ml}$ both prior to, and following, hASC differentiation down their adipogenic and osteogenic pathways. 
Fortunately, there are a number of studies that have shown that internalized NPs have no apparent deleterious effects on the morphology, cell proliferation, cell viability and differentiation efficiency of mesenchymal stem cells [9,10]. Studies involving easily functionalized polystyrene and silica NPs and derivatized magnetic NPs used for biomedical imaging, drug delivery and gene therapy have shown that their internalization into hASC does not illicit cytotoxicity $[9-11,16]$. Multiwalled carbon nanotubes incorporated into tissue engineered scaffolding were not shown to affect the viability or proliferation of hASC [17]. In addition, NPs composed of osteogenic factors have shown remarkable improvements in mesenchymal stem cell adhesion, attachment and differentiation on tissueengineered bone scaffolds [18].

The results of this study were similar to our previous research which showed that washed 20-, 50- and 80-nm Ag-NPs did not cause a decrease in viability to human epidermal keratinocytes [14]. This study determined that metallic NPs such as Ag-NPs are capable of interfering with viability assay dye and dye products through the adsorption of cell medium constituents and cytokines, which is a common effect and has been reported for several other viability assays and nanomaterials [19-21]. Based upon these findings, alamarBlue was chosen for this study due to its limited interaction with Ag-NPs. These same Ag-NPs have already been evaluated for their antibacterial efficacy in a previous study, and were found to inhibit the growth of both Gram-positive and Gram-negative bacterial strains such as E. coli, $S$. aureus, methicillin-resistant $S$. aureus and Salmonella, beginning at approximately $64 \mu \mathrm{g} /$ $\mathrm{ml}$. In the same study, $\mathrm{AgNO}_{3}$ was used as a positive control for $\mathrm{Ag}$ ions and was shown to inhibit the strains at $1.7 \mu \mathrm{g} / \mathrm{ml}$, indicating that the $64 \mu \mathrm{g} / \mathrm{ml}$ inhibitory concentration of AgNPs released at least an equivalent $1.7 \mu \mathrm{g} / \mathrm{ml}$ of $\mathrm{Ag}^{+}$. Therefore, the concentrations used in the present study were chosen to encompass the bacterial inhibitory concentration [2].

There is a general agreement that dissolved $\mathrm{Ag}$ ions are responsible for the biocidal activity that is especially pronounced against microorganisms, and this ability of Ag-NPs to slowly release these ions from their surface has made them an attractive antimicrobial additive to products. The rate of dissolution is obviously a complicated process that is dependent upon many factors including the method of Ag-NP synthesis, storage length before use, temperature, light exposure, surface functionalization and cell culture conditions [22]. The biological activity of freshly prepared and aged Ag-NPs is remarkably different due to the different amounts of released ions. For example, in a study that utilized hASC as reporter cells to analyze the effect on cell viability and morphology from the release of Ag ions over time from $50 \pm 20 \mathrm{~nm}$ citrate-stabilized Ag-NPs, it was found that $25 \mu \mathrm{g} / \mathrm{ml}$ of 3-day-old Ag-NPs were enough to cause a decrease in viability to $70 \%$, compared with a complete loss of viability for Ag-NPs first aged in water for 1-6 months [22]. Similarly, 8-nm Ag-NPs in DI water were shown to release less than $0.1 \mu \mathrm{g} / \mathrm{ml}$ of Ag ions after 1 day of incubation and up to $2 \mu \mathrm{g} / \mathrm{ml}$ after 120 days of incubation, while dissolution rates decreased with the addition of citrate stabilization or in the presence of organic matter [23]. In addition, the investigators showed that their release rates were congruent with commercial Ag-NPs stabilized with gelatin and gum Arabic, similar to previous reports [23,24].

Axiomatically, the lethal concentration of Ag-NPs will be higher than that of $\mathrm{Ag}^{+}$due to the limited release rate of $\mathrm{Ag}^{+}$from the surface of the particles. The lethal concentration of $\mathrm{Ag}^{+}$ to human cell lines such as germline stem cells, dermal fibroblasts and leukocytes is generally below $5 \mu \mathrm{g} / \mathrm{ml}$, so it is questionable that many papers report toxicity of Ag-NPs at the same concentration of reported toxicity of $\mathrm{Ag}^{+}$alone [23-27]. For example, 15-nm AgNPs synthesized in a pulsed-plasma reactor have also caused toxicity in germline stem cells at $10 \mu \mathrm{g} / \mathrm{ml}$ [24]. Controversy concerning the concentration of Ag-NP toxicity is most likely due to the Ag-NP synthesis method, whether or not the Ag-NPs are washed, and their length of storage prior to use. Ag-NP solutions may contain residual reducing agent contaminants 
that are known toxicants, a finding confirmed in this laboratory where the presence of formaldehyde in freshly synthesized Ag-NP solutions was found to be responsible for the toxicity in skin cells compared with thoroughly washed Ag-NPs [14]. The citrate stabilization of the 10- and 20-nm Ag-NPs used in this study may explain their higher lethal concentration, which is congruent with the rare studies that explicitly state that when particles are washed they result in higher lethal concentrations compared with studies that do not mention washing, generally greater than $25 \mu \mathrm{g} / \mathrm{ml}[14,22,28,29]$.

The results of this study demonstrate that exposure of 10- and 20-nm Ag-NPs either prior to differentiation, or following 14 days of hASC differentiation, resulted in no significant cytotoxicity to hASC, and minimal dose-dependent toxicity to adipogenic and osteogenic cells at $10 \mu \mathrm{g} / \mathrm{ml}$ and greater. Drawing on the results of the previous bacterial study, it is reasonable to conclude that at least $1.7 \mu \mathrm{g} / \mathrm{ml}$ was released from the Ag-NPs at the highest concentration of $100 \mu \mathrm{g} / \mathrm{ml}$ and resulted in increased cell toxicity. In each of the analyses where the hASC were first exposed to Ag-NPs and then differentiated, residual Ag-NPs remained attached to the cells throughout the 14 days of differentiation, which was confirmed by TEM. This is particularly relevant; since degradable tissue-engineered scaffolds containing Ag-NPs will shed these NPs and their ions over the course of the scaffold lifetime to impart their antimicrobial activity, and will therefore interact with the seeded progenitor cells over the course of their differentiation. Each of the progenitor, adipogenic and osteogenic cells showed cellular uptake of both 10- and 20-nm Ag-NPs, without eliciting any abnormal morphological or ultrastructural changes. Lastly, exposure to 10- and 20-nm Ag-NPs for $24 \mathrm{~h}$ did not influence the intended differentiation of the undifferentiated hASC, adipogenic or osteogenic cells, and at the antibacterial concentration of $64 \mu \mathrm{g} / \mathrm{ml}$, the Ag-NPs showed minimal toxicity.

\section{Conclusion \& future perspective}

This study showed that exposure of hASC to 10- and 20-nm Ag-NPs either prior to differentiation, or following 14 days of differentiation, resulted in no significant cytotoxicity to undifferentiated hASC. Minimal dose-dependent toxicity was observed for adipogenic and osteogenic cells at concentrations of $10 \mu \mathrm{g} / \mathrm{ml}$ and greater. The cellular uptake of AgNPs into each of the hASC, osteocytes and adipocytes did not alter their ultrastructural morphology, or influence hASC differentiation. Exposure of 10- and 20-nm Ag-NPs at concentrations up to $100 \mu \mathrm{g} / \mathrm{ml}$ did not influence hASC differentiation. Although the cell quantity limited this study to the investigation of the effect of Ag-NPs on cell viability, uptake and differentiation, these results conclude that either 10- or 20-nm Ag-NPs are suitable to incorporate into tissue-engineered scaffolds that utilize hASC.

Further studies may be conducted to ensure the biocompatibility of the Ag-NPs with hASC, including assays for hASC immunophenotype preservation or DNA stability, cytokine release, or the effects of multiple Ag-NP exposures during differentiation. These results are promising for future studies where Ag-NPs will be incorporated into tissue-engineered scaffolds. Previously evaluated scaffolding for bone graft therapies comprised of collagen, collagen coated poly( $\varepsilon$-caprolactone) and poly(L-lactic acid)/tricalcium phosphate nanofibers has been shown to increase cell-mediated calcium accretion [30-32], and would be a suitable model to evaluate Ag-NP incorporation. The addition of antimicrobial functionality, namely through the incorporation of Ag-NPs into a tissue-engineered graft scaffold would be pivotal in preventing bacterial growth and thereby minimizing infections, as well as patient and hospital costs. 


\section{Acknowledgments}

The authors would like to thank J Hardin (North Carolina State University, NC, USA) for providing assistance with the pooled human adipose-derived stem cells.

\section{References}

Papers of special note have been highlighted as:

- of interest

1. Moucha, C.; Evans, R.; Clyburn, T., et al. Orthopaedic infection prevention and control: an emerging new paradigm. Presented at: 77th Annual Meeting of the American Academy of Orthopaedic Surgeons; New Orleans, LA, USA. 9-12 March 2010;

2-. Samberg ME, Orndorff PE, Monteiro-Riviere NA. Antibacterial efficacy of silver nanoparticles of different sizes, surface conditions and synthesis methods. Nanotoxicology. 2011; 5(2):244-253. Demonstrates the efficacy of silver nanoparticles with both Gram-negative and Gram-positive bacteria. [PubMed: 21034371]

3. Morones JR, Elechiguerra JL, Camacho A, et al. The bactericidal effect of silver nanoparticles. Nanotechnology. 2005; 16:2346-2353. [PubMed: 20818017]

4. Sondi I, Salopek-Sondi B. Silver nanoparticles as antimicrobial agent: a case study on E. coli as a model for Gram-negative bacteria. J Colloid Interface Sci. 2004; 275:177-182. [PubMed: 15158396]

5. Wijnhoven SWP, Peijnenburg WJGM, Herberts CA, et al. Nano-silver - a review of available data and knowledge gaps in human and environmental risk assessment. Nanotoxicology. 2009; 3:109138 .

6. Vergroesen P-PA, Kroeze R-J, Helder MN, Smit TH. The use of poly(L-lactide-co-caprolactone) as a scaffold for adipose stem cells in bone tissue engineering: application in a spinal fusion model. Macromol Biosci. 2011; 11:722-730. [PubMed: 21400658]

7. Zhao F, Ma T. Perfusion bioreactor system for human mesenchymal stem cell tissue engineering: dynamic cell seeding and construct development. Biotechnol Bioeng. 2005; 91(4):482493.

8. Zuk PA, Zhu M, Ashjian P, et al. Human adipose tissue is a source of multipotent stem cells. Mol Cell Biol. 2002; 13(12):4279-4295.

9. Chung T-H, Wu S-H, Yao M, et al. The effect of surface charge on the uptake and biological function of mesoporous silica nanoparticles in 3T3-L1 cells and human mesenchymal stem cells. Biomaterials. 2007; 28:2959-2966. [PubMed: 17397919]

10. Huang D-M, Chung T-H, Hung Y, et al. Internalization of mesoporous silica nanoparticles induces transient but not sufficient osteogenic signals in human mesenchymal stem cells. Tox Appl Pharma. 2008; 231:208-215.

11. Lewin M, Carlesso N, Tung C-H, et al. Tat peptide-derivatized magnetic nanoparticles allow in vivo tracking and recovery of progenitor cells. Nature Biotech. 2000; 18:410-414.

12. Bernacki SH, Wall ME, Loboa EG. Isolation of human mesenchymal stem cells from bone and adipose tissue. Methods Cell Biol. 2008; 86:257-278. [PubMed: 18442651]

13. Wall ME, Bernacki SH, Loboa EG. Effects of serial passaging on the adipogenic and osteogenic differentiation potential of adipose-derived human mesenchymal stem cells. Tissue Eng. 2007; 13(6):1291-1298. [PubMed: 17518709]

14-. Samberg ME, Oldenburg SJ, Monteiro-Riviere NA. Evaluation of silver nanoparticle toxicity in skin in vivo and keratinocytes in vitro. Environ Health Perspect. 2010; 118(3):407-413. Depicts the toxicity of silver nanoparticles of different sizes and synthesis methods in vitro and in vivo. [PubMed: 20064793]

15. Giannoudis PV, Dinopoulos H, Tsiridis E. Bone substitutes: an update. Injury. 2005; 36S:S20S27. [PubMed: 16188545]

16. Lorenz MR, Holzapfel V, Musyanovych A, et al. Uptake of functionalized, fluorescent-labeled polymeric particles in different cell lines and stem cells. Biomaterials. 2006; 27 (14):2820-2828. [PubMed: 16430958] 
17. McCullen SD, Stevens DR, Roberts WA, et al. Characterization of electrospun nanocomposite scaffolds and biocompatibility with adipose-derived human mesenchymal stem cells. Int $\mathbf{J}$ Nanomedicine. 2007; 2:253-263. Incorporation of multiwalled carbon nanotubes into electrospun nanofiber mats produces porous nanocomposite structures with enhanced mechanical and electrical properties. [PubMed: 17722553]

18. Lock J, Liu H. Nanomaterials enhance osteogenic differentiation of human mesenchymal stem cells similar to a short peptide of BMP-7. Int J Nanomedicine. 2011; 6:2769-2777. [PubMed: 22114505]

19. Monteiro-Riviere NA, Inman AO. Challenges for assessing carbon nanomaterial toxicity to the skin. Carbon. 2006; 44(6):1070-1078.

20. Monteiro-Riviere NA, Inman AO, Zhang LW. Limitations and relative utility of screening assays to assess engineered nanoparticle toxicity in a human cell line. Tox Appl Pharma. 2009; 234:222235.

21. Zhang LW, Zeng L, Barron AR, Monteiro-Riviere NA. Biological interactions of functionalized single-wall carbon nanotubes in human epidermal keratinocytes. Int J Toxicol. 2007; 26:103-113. [PubMed: 17454250]

22. Kittler S, Greulich C, Diendorf J, Köller M, Epple M. Toxicity of silver nanoparticles increases during storage because of slow dissolution under release of silver ions. Chem Mater. 2010; 22:4548-4554.

23. Liu J, Hurt R. Ion release kinetics and particle persistence in aqueous nano-silver colloids. Environ Sci Technol. 2010; 44:2169-2175. [PubMed: 20175529]

24. Braydich-Stolle L, Hussain S, Schlager J, Hofmann MC. In vitro cytotoxicity of nanoparticles in mammalian germ line stem cells. Toxicol Sci. 2005; 88:412-419. [PubMed: 16014736]

25. Hidalgo E, Dominguez C. Study of cytotoxicity mechanisms of silver nitrate in human dermal fibroblasts. Information Toxicol Lett. 98(3):169-179.

26. Jansson G, Harms-Ringdahl M. Stimulating effects of mercuric and silver ions on the superoxide anion production in human polymorphonuclear leukocytes. Free Radical Res Commun. 1993; 18:87-98. [PubMed: 8386687]

27. Park E-J, Yi J, Kim Y, Choi K, Parl K. Silver nanoparticles induce cytotoxicity by a Trojan-horse type mechanism. Toxicol In Vitro. 2010; 24:872-878. [PubMed: 19969064]

28. Ahamed M, Karns M, Goodson M, et al. DNA damage response to different surface chemistry of silver nanoparticles in mammalian cells. Toxicol Appl Pharmacol. 2008; 233 (3):404-410. [PubMed: 18930072]

29. Liu W, Wu Y, Wang C, et al. Impact of silver nanoparticles on human cells: effect of particle size. Nanotoxicology. 2010; 4(3):319-330. [PubMed: 20795913]

30. Sumanasinghe RD, Bernacki SH, Loboa EG. Osteogenic differentiation of human mesenchymal stem cells in collagen matrices: effect of uniaxial cyclic tensile strain on bone morphogenetic protein (BMP-2) mRNA expression. Tissue Eng. 2006; 12(12):3459-3465. [PubMed: 17518682]

31. Haslauer CM, Moghe AK, Osborne JA, Gupta BS, Loboa EG. Collagen-PCL sheath-core bicomponent electrospun scaffolds increase osteogenic differentiation and calcium accretion of human adipose-derived stem cells. J Biomat Sci Polym Ed. 2011; 22(13):1695-1712.

32-. McCullen SD, Zhu Y, Bernacki SH, et al. Electrospun composite poly(L-lactic acid)/ tricalcium phosphate scaffolds induce proliferation and osteogenic differentiation of human adipose-derived stem cells. Biomed Mater. 2009; 4:035002. Suggests that local environment cues provided by the biochemical nature of the scaffold can accelerate the overall osteogenic differentiation of human adipose-derived stem cells and encourage rapid ossification. [PubMed: 19390143]

\section{Websites}

101. CDC. [Accessed 25 August 2011] Surgical Site Infection. www.cdc.gov/ncidod/dhqp/pdf/ guidelines/SSI_tagged.pdf

102. The project on emerging nanotechnologies. Woodrow Wilson International Center for Scholars; Washington, DC, USA: www.nanotechproject.org/inventories/consumer/analysis_draft/ 
103. Silver: Integrated Risk Information System. United States Environmental Protection Agency; www.epa.gov/iris/ 


\section{Executive summary}

- Exposure of 10- and 20-nm silver (Ag)-nanoparticles (NPs) either prior to differentiation, or following 14 days of human adipose-derived stem cell (hASC) differentiation, resulted in no significant cytotoxicity to hASC. Minimal dose-dependent toxicity was observed for adipogenic and osteogenic cells at concentrations of $10 \mu \mathrm{g} / \mathrm{ml}$ and greater.

- Exposure of 10- and 20-nm Ag-NPs either prior to differentiation, or following 14 days of hASC differentiation, resulted in cellular uptake into each of the progenitor, adipogenic and osteogenic cells, without eliciting ultrastructural alterations.

- Exposure of 10- and 20-nm Ag-NPs for $24 \mathrm{~h}$ did not influence the intended differentiation of the cells.

- Ag-NPs are suitable for tissue-engineered scaffolding incorporation at the antibacterial concentration of $64 \mu \mathrm{g} / \mathrm{ml}$. 


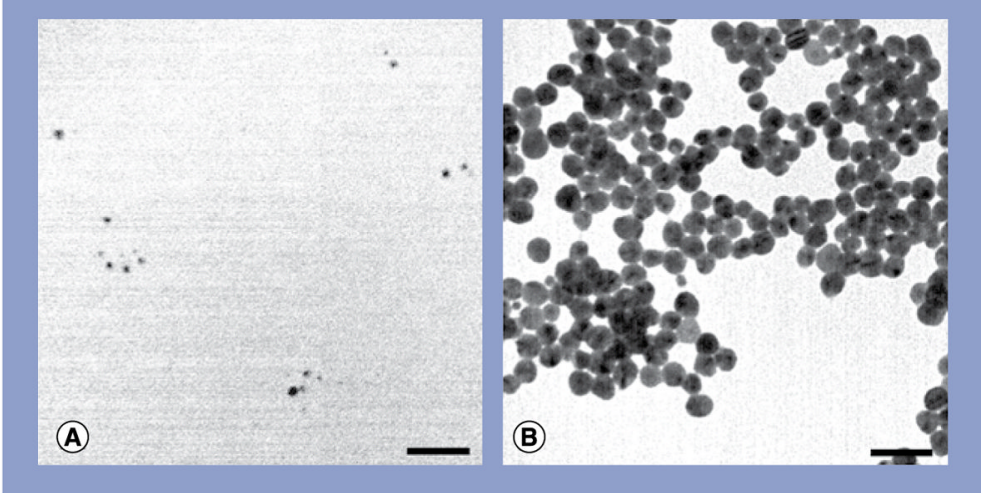

Figure 1. Transmission electron micrographs of silver nanoparticles (A) $10 \mathrm{~nm}$ and (B) $20 \mathrm{~nm}$. Scale bars: $50 \mathrm{~nm}$. 

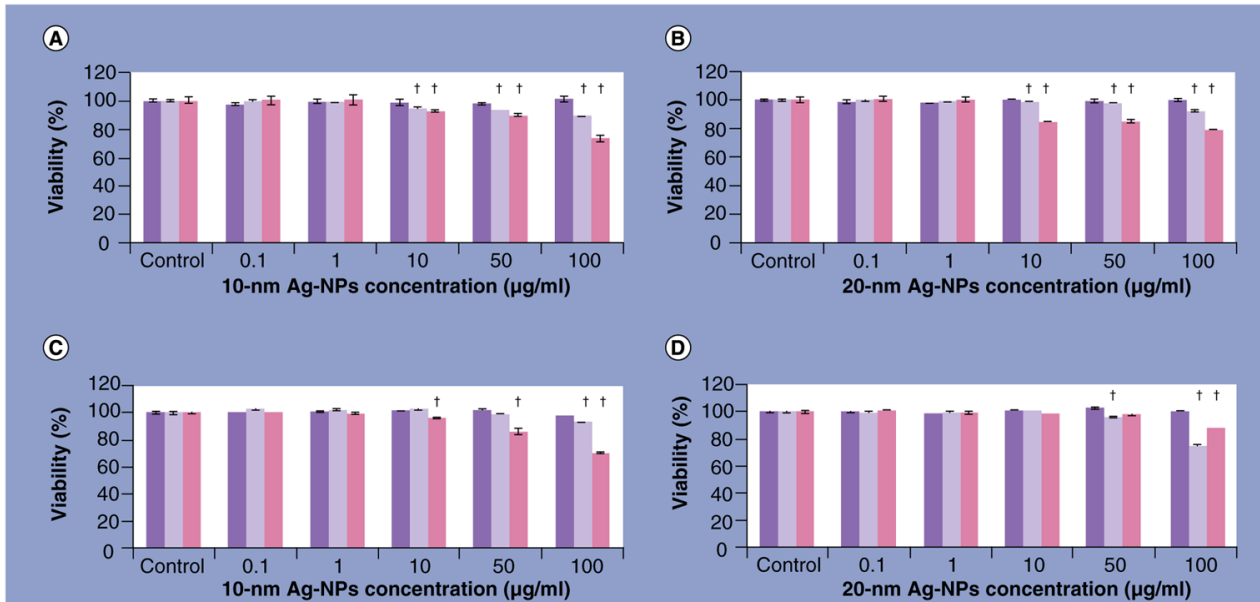

(D)

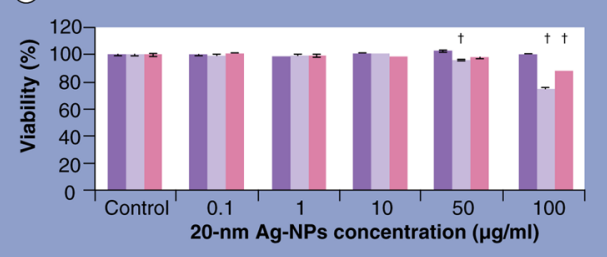

- Progenitor $=$ Osteogenic $=$ Adipogenic

Figure 2. alamarBlue $^{\circledR}$ viability of undifferentiated and differentiated human adipose-derived stem cells exposed to silver nanoparticles

alamarBlue viability of undifferentiated human adipose-derived stem cells, treated with either (A) 10 or (B) $20 \mathrm{~nm}$ Ag-NPs and then differentiated for 14 days; human adiposederived stem cells differentiated for 14 days and then treated with (C) 10 or (D) 20-nm AgNPs.

${ }^{\dagger}$ Significant differences $(\mathrm{p}<0.05)$ in viability were assessed by multiple comparisons between concentrations, per cell pathway.

Ag: Silver; NP: Nanoparticle. 


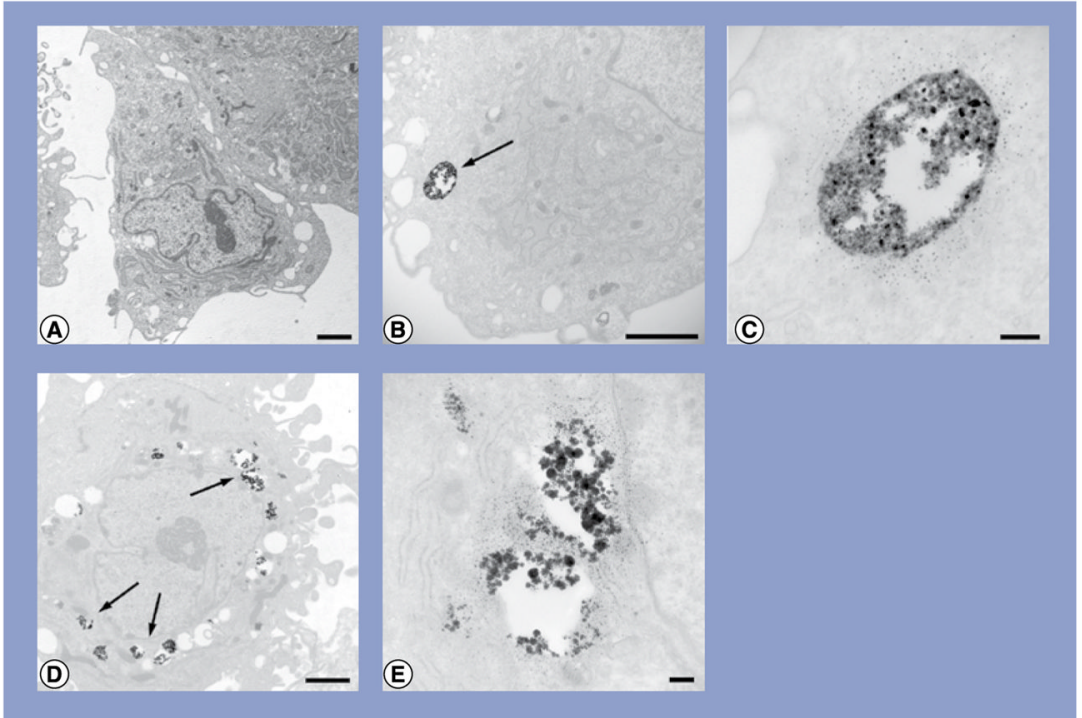

Figure 3. Transmission electron micrographs of human adipose-derived stem cells exposed to either 10- or 20-nm silver nanoparticles at $10 \mu \mathrm{g} / \mathrm{ml}$

(A) Human adipose-derived stem cells (hASCs); (B) hASC treated with 10-nm silver nanoparticles (Ag-NPs); (C) higher magnification of vacuole containing Ag-NPs from (B); (D) hASC treated with 20-nm Ag-NPs and (E) higher magnification of vacuoles containing Ag-NPs from (D). Scale bars: $2 \mu \mathrm{m}$ (A, B \& D); scale bars: $200 \mathrm{~nm}$ (C \& E). Arrows denote agglomerates of Ag-NPs. 


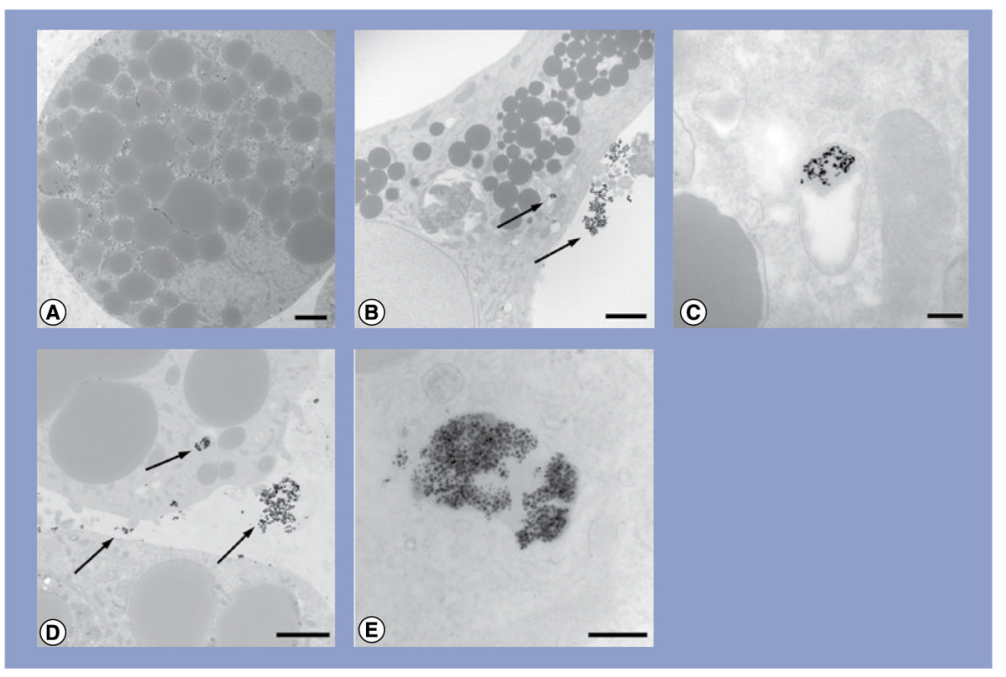

Figure 4. Transmission electron micrographs of adipogenic cells exposed to either 10- or 20-nm silver nanoparticles at $10 \mu \mathrm{g} / \mathrm{ml}$

(A) Adipogenic cell showing spherical lipid accumulation; (B) adipogenic cell treated with 10-nm silver nanoparticles (Ag-NPs); (C) higher magnification of the vacuole containing Ag-NPs from (B); (D) adipogenic cell treated with 20-nm Ag-NPs and (E) higher magnification of the vacuole containing Ag-NPs from (D). Scale bars: $2 \mu \mathrm{m}$ (A, B \& D); scale bars: 200 nm (C \& E). Arrows denote agglomerates of Ag-NPs. 


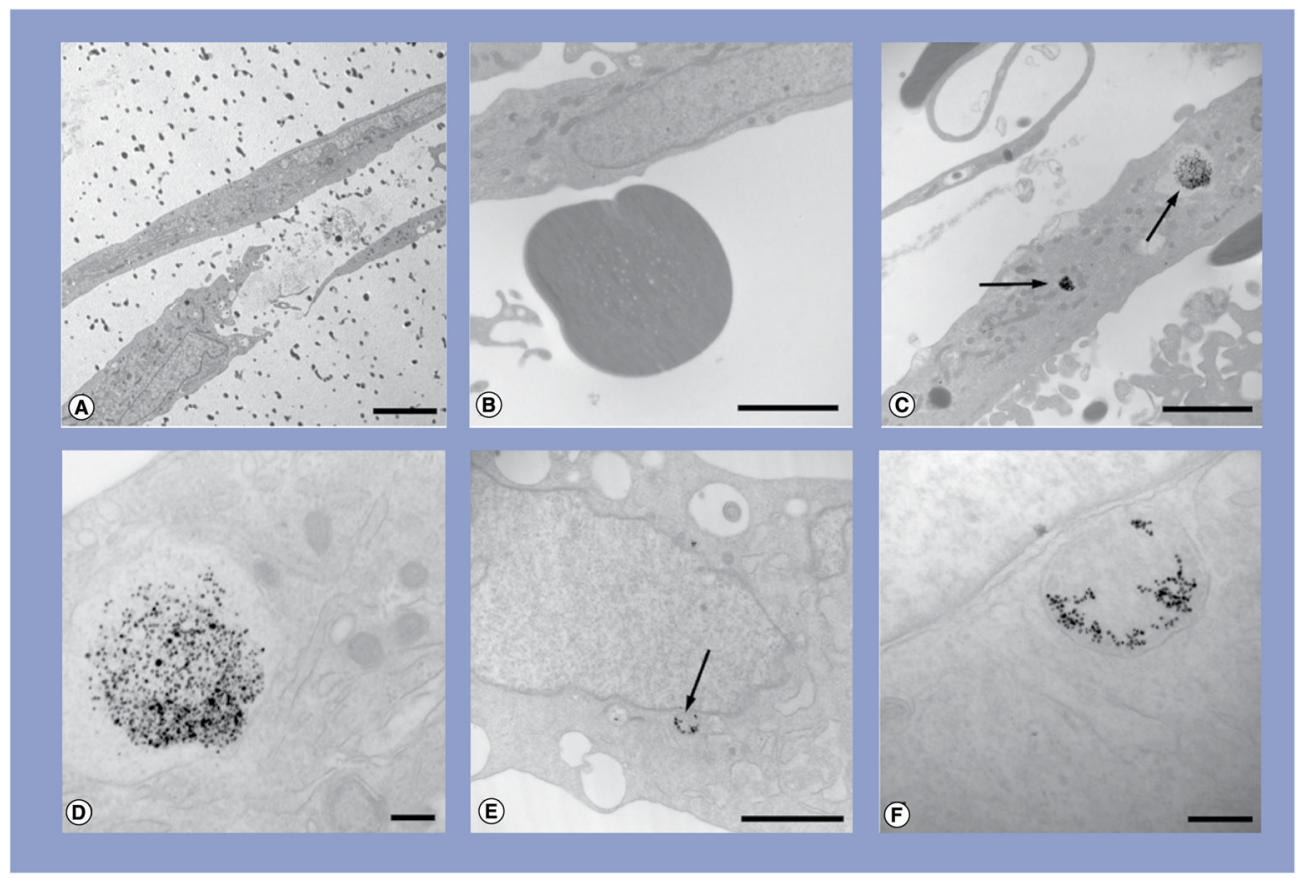

Figure 5. Transmission electron micrographs of osteogenic cells exposed to either 10- or 20-nm silver nanoparticles at $10 \mu \mathrm{g} / \mathrm{ml}$

(A) Osteogenic cell showing extracellular calcium accretion (dark dots); (B) higher magnification of accreted calcium; (C) osteogenic cell treated with 10-nm silver nanoparticles (Ag-NPs); (D) higher magnification of the vacuole containing Ag-NPs from (C); (E) adipogenic cell treated with 20-nm Ag-NPs and (F) higher magnification of the vacuole containing Ag-NPs from (E). Scale bar: $5 \mu \mathrm{m}$ (A); scale bars: $2 \mu \mathrm{m}$ (B, C \& E); scale bars: $200 \mathrm{~nm}$ (D \& F). Arrows denote agglomerates of Ag-NPs. 

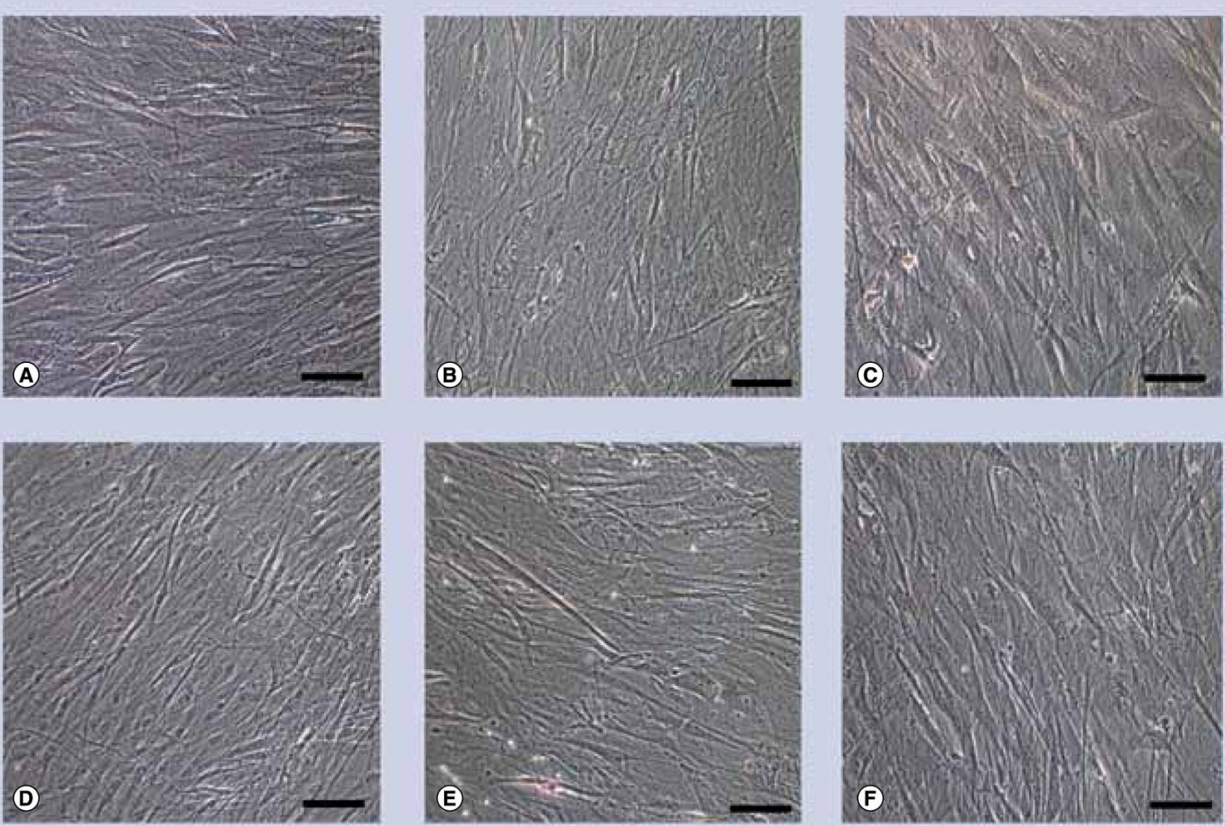

Figure 6. Light micrographs of undifferentiated human adipose-derived stem cells exposed to either 10- or 20-nm silver nanoparticles at $10 \mu \mathrm{g} / \mathrm{ml}$

(A-C) No stain for either calcium accretion with Alizarin Red S or for (D-F) lipid accumulation with Oil Red O. Human adipose-derived stem cells (A \& D) exposed to 10-nm silver nanoparticles (Ag-NPs) (B \& E), or 20-nm Ag-NPs (C \& F). Scale bars: 0.1 mm. 

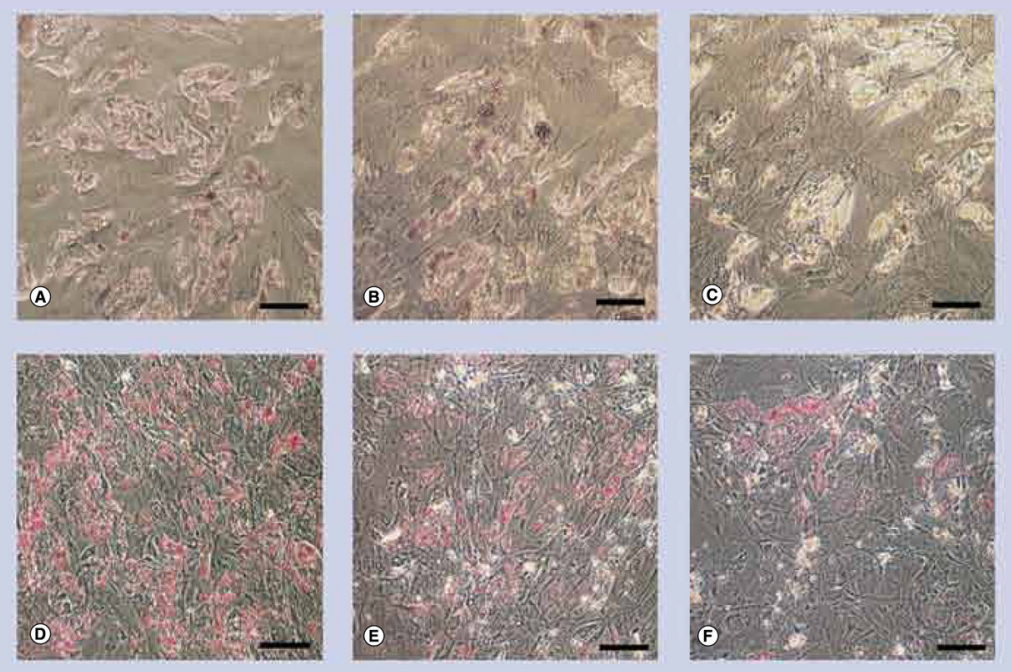

Figure 7. Light micrographs of adipogenic cells exposed to either 10- or 20-nm silver nanoparticles at $10 \mu \mathrm{g} / \mathrm{ml}$. (A-C)

No stain for calcium accretion with Alizarin Red S and (D-F) strong staining for lipid accumulation with Oil Red O (cherry red spots). Control cells (A \& D) exposed to 10-nm silver nanoparticles (Ag-NPs) (B \& E), or 20-nm Ag-NPs (C \& F). Scale bars: $0.1 \mathrm{~mm}$. 

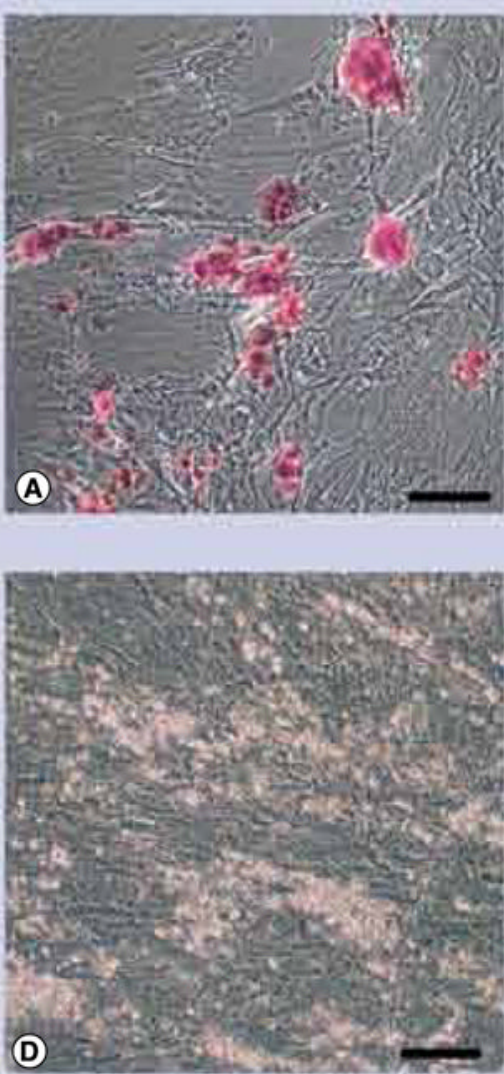
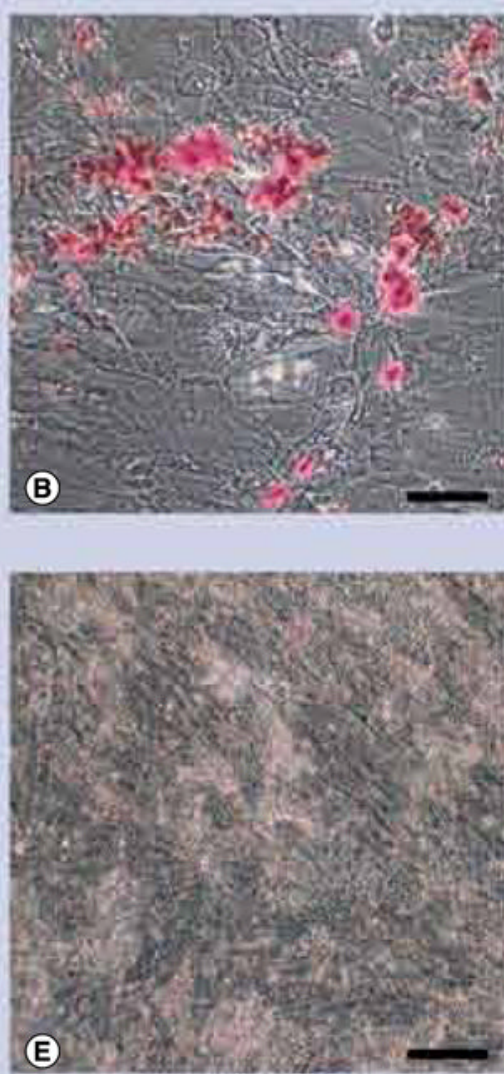
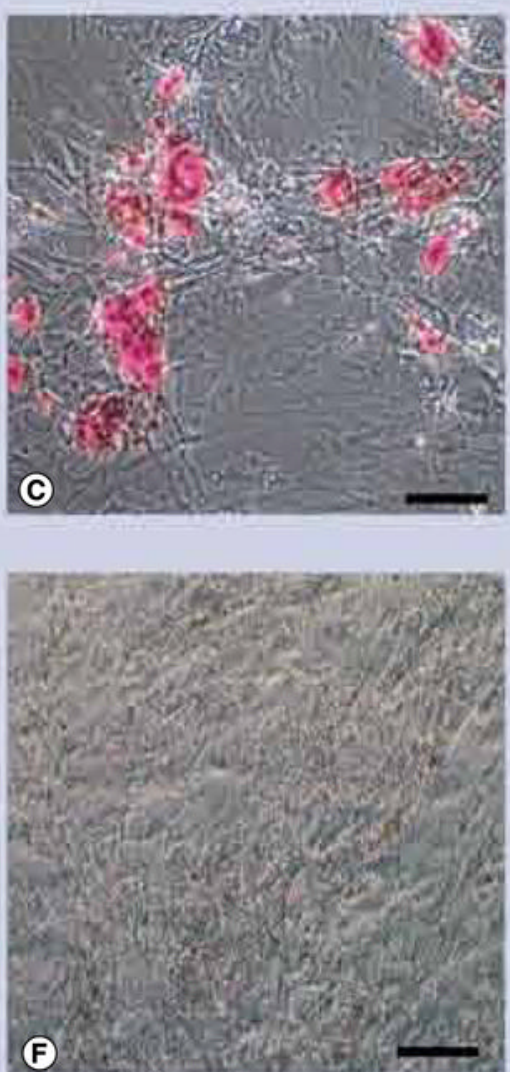

Figure 8. Light micrographs of osteogenic cells exposed to either 10- or 20-nm silver nanoparticles at $10 \mu \mathrm{g} / \mathrm{ml}$. (A-C)

exhibits strong staining for calcium accretion with Alizarin Red S (red spots) but none for (D-F) lipid accumulation with Oil Red O. Control cells (A \& D) exposed to 10-nm silver nanoparticles (Ag-NPs) (B \& E) or 20-nm Ag-NPs (C \& F). Scale bars: $0.1 \mathrm{~mm}$. 


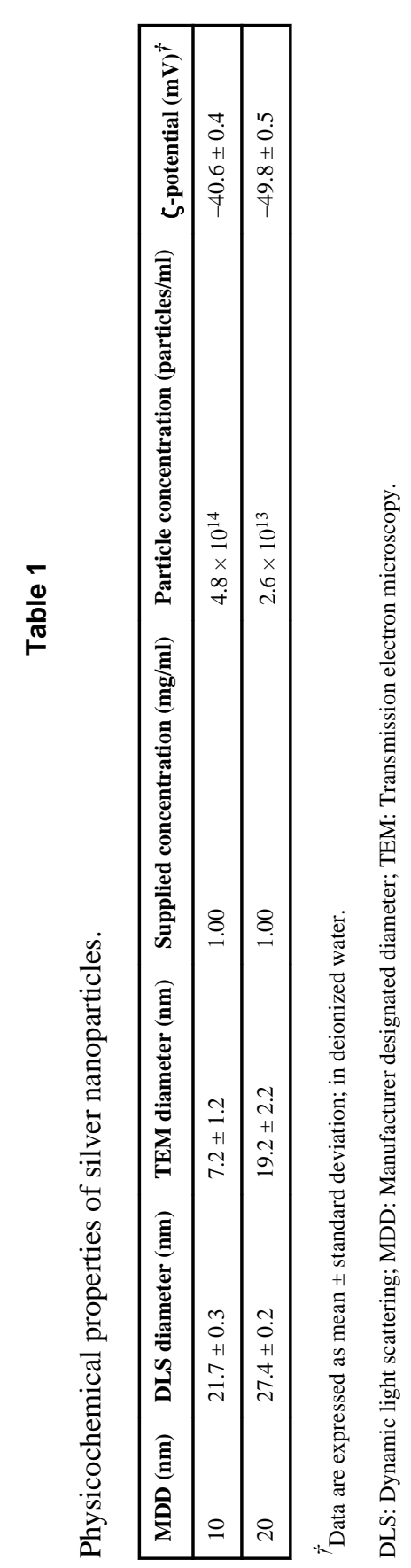

Nanomedicine (Lond). Author manuscript; available in PMC 2012 October 17. 